\title{
'It's All Done With Mirrors': V.S. Ramachandran and the Material Culture of Phantom Limb Research
}

\author{
KATJA GUENTHER * \\ History Department, 129 Dickinson Hall, Princeton University, Princeton, NJ 08544, USA
}

\begin{abstract}
This article examines the material culture of neuroscientist Vilayanur S. Ramachandran's research into phantom limbs. In the 1990s Ramachandran used a 'mirror box' to 'resurrect' phantom limbs and thus to treat the pain that often accompanied them. The experimental success of his mirror therapy led Ramachandran to see mirrors as a useful model of brain function, a tendency that explains his attraction to work on 'mirror neurons'. I argue that Ramachandran's fascination with and repeated appeal to the mirror can be explained by the way it allowed him to confront a perennial problem in the mind and brain sciences, that of the relationship between a supposedly immaterial mind and a material brain. By producing what Ramachandran called a 'virtual reality', relating in varied and complex ways to the material world, the mirror reproduced a form of psycho-physical parallelism and dualistic ontology, while conforming to the materialist norms of neuroscience today.
\end{abstract}

Keywords: Material culture, Mirrors,

Neuroscience, Phantom limb pain, Virtual reality, Sigmund Freud

In the early 1990s, an advertisement appeared in San Diego newspapers seeking amputees for a range of clinical experiments. Something about the advertisement hinted at the occult; it talked of parts of the body that couldn't be seen, and yet remained irrefutable presences to their owners; these were 'phantoms'. ${ }^{1}$ Phantom limbs had long been a recognised medical phenomenon. People who had lost a limb would often claim that they could continue to feel their amputated arm or leg. Some would even act as if it were still present, gesticulating with the phantom while speaking. The strange unreal quality of the phantom, however, ensured that it only rarely achieved serious scrutiny in medical circles. Although a number of distinguished mainstream physicians chose these phantoms as a topic of study (doctors like the sixteenth-century French surgeon Ambroise Paré or the prominent American neurologist Silas Weir Mitchell, who coined the term 'phantom

* Email address for correspondence: kguenthe@princeton.edu

I would like to thank the following people for their helpful comments and suggestions: Edward Baring, Volker Hess, Leor Katz, Sophie Ledebur, the participants of the 'Soul Catchers' Workshop at Princeton in February 2014, the participants of the 'Pathology and Plasticity' Workshop at Berkeley in April 2014, and the three anonymous reviewers for Medical History.

${ }^{1}$ V.S. Ramachandran and D. Rogers-Ramachandran, 'Synaesthesia in Phantom Limbs Induced with Mirrors', Proceedings of the Royal Society of London B, 263 (1996), 377-86: 379. They had recruited patients in a number of ways: some had been referred through the orthopaedics department at the UCSD medical school; others they found through contacting local prosthesis manufacturers in San Diego. 
limb' in 1871), as scholars have noted, an important part of the phenomenon's history has in fact been its marginality. ${ }^{2}$ Aura Satz has recently shown that in the second half of nineteenth-century America, when amputees gained visibility due both to accidents in an industrialising society and to the legacy of the Civil War, discussions about phantom limbs were closely linked to explorations of the supernatural within the cultural practice of spiritual séances, which aimed to recover lost bodies. Even the term 'phantom' tapped into the culture of phantasmagoria (a form of cinema using a modified magic lantern) of the time. ${ }^{3}$

By the 1990s the spiritualist context of the phantom limb was a thing of the past, yet the medical profession continued to consider phantoms as clinical curiosities. It is telling that the term 'phantom' stuck. The syndrome and the pain associated with it remained difficult to reconcile with mainstream medicine; like a ghost it was hard to prove, pindown, or analyse, perhaps even believe. Indeed amputees, especially of the statistically less prevalent upper extremities, have been relatively marginalised within medicine. Up to that time the most important and widespread treatment for amputees involved prostheses, which were predominantly 'passive', that is simple and inert, and the rejection rate was high. ${ }^{4}$

Times, however, were changing, not least for the amputees themselves. In 1990 Congress had passed the Americans Disabilities Act (ADA) with an overwhelming majority. The legislation attracted considerable public attention and substantial praise from disability activists. Most important to the latter were the provisions for protection from discrimination in the workplace, spelled out in detail in Title I of the document. ${ }^{5}$ The Act helped spur the production of body-powered and electric prostheses, the development of which only kicked off properly after 1990 and brought a much-needed excitement to the field. ${ }^{6}$

${ }^{2}$ Ambroise Paré, Oeuvres complètes d'Ambroise Paré, Vol. 2, ed. J.F. Malgaigne (Paris: Baillère, 1840-1). S. Weir Mitchell, 'Phantom limbs', Lippincott's Magazine of Popular Literature and Science, 8 (1871), 5639. Aura Satz, "The conviction of its existence": Silas Weir Mitchell, phantom limbs and phantom bodies in neurology and spiritualism', in L. Salisbury and A. Shail (eds), Neurology and Modernity: A Cultural History of Nervous Systems, 1800-1950 (New York: Palgrave Macmillan, 2010), 113-29. See also Douglas B. Price and Neil J. Twombly, The Phantom Limb Phenomenon: A Medical Folkloric, and Historical Study: Texts and Translations of 10th to 20th Century Accounts of the Miraculous Restoration of Lost Body Parts (Washington DC: Georgetown University Press, 1978). Lisa Herschberg, “ “True Clinical Fictions:' Medical and Literary Narratives from the Civil War Hospital', Culture, Medicine, and Psychiatry, 19, 2 (1995), 183-205. For a stimulating history and sociology of the phantom limb see Cassandra Crawford, Phantom Limb: Amputation, Embodiment, and Prosthetic Technology (New York: New York University Press, 2014).

${ }^{3}$ Satz, op. cit. (note 2), 114.

4 The rejection rate since the 1980 s has been determined as $39 \%$. See Elaine A. Biddiss and Tom T. Chau, 'Upper limb prosthesis use and abandonment: A survey of the last 25 years', Prosthetics and Orthotics International (2007), percentage of 250. On the prevalence of upper limb amputations as opposed to lower limb, see, for example, G. Täger and D. Nast-Kolb, 'Amputationen und Prothesenversorgung der oberen Extremität', Der Chirurg, 71, 6 (2000), 727-42, which gives data for the US and Germany. The history of prostheses has primarily been told in a war or post-war context, see, for example, Sabine Kienitz, Beschädigte Helden: Kriegsinvalidität und Körperbilder 1914-23 (Paderborn: Schnöingh, 2008). David Harley Serlin, Replaceable You: Engineering the Body in Postwar America (Chicago, IL: University of Chicago Press, 2004), ch. 1. Laurann Figg and Jane Farrell-Beck, 'Amputation in the Civil War: Physical and Social Dimensions', Journal of the History of Medicine and Allied Sciences, 48 (1993), 454-75. For a discussion of 'phantom-prosthetic relations', see the chapter in Crawford, op. cit. (note 2).

${ }^{5}$ Ruth Colker, The Disability Pendulum: The First Decade of the Americans with Disabilities Act (New York: New York University Press, 2005). See here also for the backlash that followed the high hopes associated with the 1990 Act.

${ }^{6}$ This also improved the reputation of amputation. By 2000, the developments in prosthesis helped reconceptualise amputation surgery as 'constructive', Crawford, op. cit. (note 2), 213. 
The San Diego advertisements suggested that the conventional dismissal of the phantom-limb phenomenon to the fringes of academic medicine might be changing too; the condition was gaining respectability. The experimental set-up described by the advertisements had all the markings of reputable science. Above all, the name of the chief researcher reassured the participants: the advertisements had been placed by the neurologist Vilayanur S. Ramachandran, director of the San Diego Center for Brain and Cognition at the University of California. Ramachandran's increasing prominence within and outside the field of neuroscience helped legitimise the project. At the time, Ramachandran was not just a household name within the confines of the University or even the South Californian city; his imaginative experiments and novel therapeutic regimes were beginning to gain traction in the global world of neuroscience, as well as in the popular imagination. Originally trained as a physician in Madras, India, Ramachandran earned a $\mathrm{PhD}$ in neuroscience from the University of Cambridge. Not long after the advertisement was placed, Newsweek named him a member of the 'Century Club', one of the 'hundred most prominent people to watch' in the twenty-first century. He has since held prestigious fellowships at All Souls College, Oxford, and Stanford University. In 2011, Time magazine named him, alongside Barack Obama and Justin Bieber, as one of the 100 most influential people in the world. ${ }^{7}$

Ramachandran confronted the previously marginal status of 'phantom limbs' directly. He was convinced that 'far from being mere oddities, [phantom limbs] illustrate certain important principles underlying the functional organisation of the normal human brain' ${ }^{8}$ Ramachandran made phantoms respectable, because he argued that they were not peripheral to the general work of neuroscientists. On the contrary, they shed light on neurological processes central to all mental activity. The study of phantom limbs promised to help neurologists explain a wide variety of neurological phenomena.

At first glance, Ramachandran's experiments seem to mark the integration of the phantom limb into mainstream biomedicine, the triumph of the somaticist model over the occult. But, although Ramachandran presented his work as a wholly materialist explanation for phantom limbs, if we examine the material culture of his experiments, it becomes clear that he did not fully expunge the vexing immateriality of the phantom. Rather, his experiments depended upon a device that produced a similar unreal presence, haunting the real world. When Ramachandran came to see the phantom as the key for understanding all mental processes, that device would become his favoured metaphor for understanding the mind, for, while Ramachandran gave no credence to talk of 'ghosts' or 'phantoms', he did talk, almost obsessively, about 'mirrors'.

\section{Material Culture in the History of Science}

To approach the study of phantom limbs through the material culture of Ramachandran's lab might seem a foolhardy endeavour. The main works of scholarship on material culture in the history of science have examined the ways in which machines and experimental apparatuses have been used to study material objects. Take, for instance, Peter Galison's Image and Logic, a book about the 'machines of physics', such as the bubble chamber

\footnotetext{
${ }^{7}$ Though I have structured this paper around Ramachandran, I am less interested in the status, influence and critiques of his work than in the ways in which it provides a fascinating and clear example of how mirrors have functioned in the 'mind sciences'.

${ }^{8}$ V.S. Ramachandran et al., 'Illusions of body image: what they reveal about human nature', in R. Linas and P. Churchland (eds), The Mind-Brain Continuum (Cambridge, MA: MIT Press): 29-60: 30.
} 
or the Geiger counter. ${ }^{9}$ These machines allowed physicists to study the 'microworld': the smallest forms of matter such as electrons, photons, protons and quarks, because the physical properties of those particles interacted with the machines to produce visible representations of their activity. For instance bubble chambers were constructed in such a way that subatomic particles would produce paths of small bubbles in superheated liquid hydrogen. Galison's machines could mediate between scientific objects and the knowledge about these objects, because they could interact materially with those objects. ${ }^{10}$

Hans-Jörg Rheinberger's approach differs from Galison's in many ways. He posits a greater instability both at the level of machines (elements of his 'experimental systems') and the scientific objects (his 'epistemic things'). By virtue of their capacity for 'differential reproduction', experimental systems were capable of creating unforeseeable scientific events; they were 'machine[s] for making the future'. ${ }^{11}$ But as for Galison, Rheinberger's machines could help produce epistemic things because they shared physical properties. His main example, the in vitro synthesis of proteins, makes this clear. Of course, these were 'things embodying concepts' ${ }^{12}$; the transfer RNA that emerged from 'soluble RNA' within Rheinberger's experimental system embodied amongst others Francis Crick's 'adaptor hypothesis', which introduced the language of information transfer. But as Rheinberger himself asserts, his 'epistemic things' were 'material entities or processes - physical structures, chemical reactions, biological functions'. ${ }^{13}$ In these two canonical accounts of material culture, then, we see a commonality between the apparatuses used by scientists and the objects studied; it seems that material culture is useful for studying material things. If this is true, then phantom limbs would present a troubling counterexample, because they are defined precisely by their lack of material presence.

In such a situation, one might be tempted to turn to Bruno Latour for a solution. In his Actor-Network-Theory (ANT), Latour sets aside any ontological distinctions between different 'actors'. Arguing more broadly against a 'sociology of the social' which assumes the existence of a society as a distinct entity that provides a context framing the activities taking place within it, he proposes instead a 'sociology of associations', a study of relations between things that are not social themselves. ${ }^{14}$ In this context, 'things' are broadly defined as those entities that have an effect, that 'act', with any preconceptions about their ontological status (whether they be matter, ideas or spirits) put to one side. Before Pasteur knew what the entity was that he observed in a series of laboratory trials on lactic acid fermentation, he knew what it did. ${ }^{15}$ A marker of this approach is that it includes non-human, as well as human, actors. This, Latour points out, is not to establish 'some absurd "symmetry between humans and non-humans", but rather to avoid the assumption of an a priori asymmetry or, indeed, any pre-configured relationship between 'actors' ${ }^{16}$

\footnotetext{
${ }^{9}$ Peter Galison, Image and Logic: A Material Culture of Microphysics (Chicago, IL: University of Chicago Press, 1997), xvii.

${ }^{10}$ Galison, op. cit. (note 9), xviii.

${ }^{11}$ Hans-Jörg Rheinberger, Toward a History of Epistemic Things: Synthesizing Proteins in the Test Tube (Stanford, CA: Stanford University Press, 1997), 33. Note that here experimental systems and epistemic things are mutually constitutive.

12 Rheinberger, Ibid., 9.

13 Rheinberger, Ibid., 28.

${ }^{14}$ Bruno Latour, Reassembling the Social: An Introduction to Actor-Network Theory (Oxford: Oxford University Press, 2005).

15 Bruno Latour, Pandora's Hope: Essays on the Reality of Science Studies (Cambridge, MA: Harvard University Press, 1999), 119. The entity was later called 'yeast'.

${ }^{16}$ Latour, op. cit. (note 14), 76.
} 
In doing so, Latour embraces a broad notion of the 'material', one that goes beyond the Cartesian understanding of matter: 'the research field should be made wide open to begin with and it cannot be opened if the difference between human action and material causality is maintained as adamantly as Descartes's distinguished mind from matter (res extensa from the res cogitans) '. ${ }^{17}$ For Latour then, the material culture of phantom limb research would not have any privileged or problematic status, because we would dispense from the start with any presuppositions that would oppose an immaterial phantom to the material instruments that are supposed to study it.

We might, however, hesitate before following Latour down the road to such ontological flattening. Certainly his Actor-Network-Theory has had its critics, and they have often focused on the parity he attributes to different objects. ${ }^{18}$ But here I would like to put aside Latour's work because he makes the solution too easy. Or rather, his theory tends to efface rather than solve what is an interesting historical problem. When we retain, if only provisionally, the ontological distinction between the phantom and the physical, it focuses our attention on the specific ways in which scientists have constructed experimental apparatuses to study those things that escape the traditional bounds of what is considered scientific. It asks us to consider how in the framework of somatic medicine, neuroscientists might still aim and be able to catch souls.

\section{Treating Phantom Limbs: The Mirror Box}

The most prominent mirror in Ramachandran's work was at the centre of his famous 'mirror' or 'virtual reality box therapy'. The device was constructed by placing a mirror vertically in the middle of a wooden box (Figure 1). The patient would then place her healthy arm into one side, and the phantom into the other. The top and front side of the box were open, which allowed the patient to look into the box, but for the purposes of the treatment she had to cock her head to one side such that she could see the mirror from the side of the box with the healthy arm. Next, she was asked to move her healthy arm around until its mirror image 'superimposed the felt position of the phantom'. ${ }^{19}$ If working properly, when patients performed 'mirror-symmetrical movements' they would see their phantom arm 'resurrected' ${ }^{20}$

The mirror box procedure was conceived of as a therapy and confronted a central feature of the phantom experience: pain. The ways in which phantom limb patients experienced their missing limb varied greatly across patients, as much as the cause could vary, ranging from crush injuries suffered in car or motorcycle accidents, to melanoma infiltrating the nervous structures of the arm, to self-inflicted amputation. ${ }^{21}$ But for many if not most of the amputees, their phantom limbs were a burden, a source of pain and anxiety. That pain might be described as 'burning, cramping, crushing or lancinating' and could be intermittent or permanently felt. ${ }^{22}$ Through the use of his 'mirror box' many of Ramachandran's patients experienced dramatic improvement in their conditions;

\footnotetext{
${ }^{17}$ Latour, Ibid., 85.

18 For example, Simon Schaffer who accuses him of regressing into 'hylozoism', the doctrine that attributes life to matter. Simon Schaffer, 'The Eighteenth Brumaire of Bruno Latour', Studies in History and Philosophy of Science, 22, 1 (1991), 174-92: 182.

19 Ramachandran and Rogers-Ramachandran, op. cit. (note 1), 380.

${ }^{20}$ Ramachandran et al., op. cit. (note 8), 31.

${ }^{21}$ Ramachandran and Rogers-Ramachandran, op. cit. (note 1), 379ff.

22 V.S. Ramachandran and Eric Altschuler, 'The Use of Visual Feedback, in particular Mirror Visual Feedback, in Restoring Brain Function’, Brain, 132 (2009), 1693-710: 1694.
} 


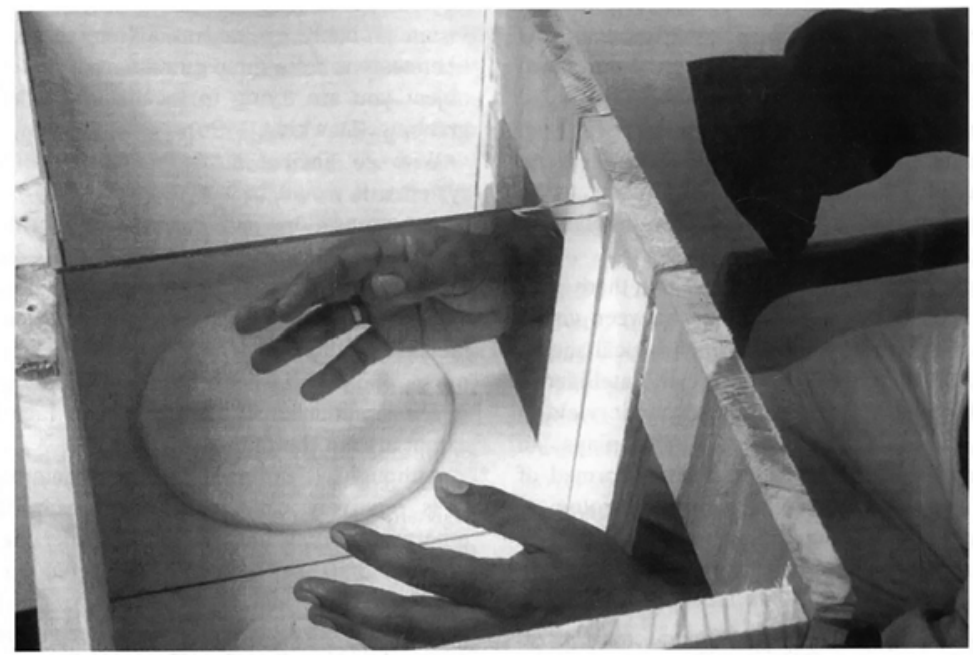

Figure 1: V.S. Ramachandran's mirror box. V.S. Ramachandran and William Hirstein, 'The Perception of Phantom Limbs: The D.O. Hebb Lecture', Brain, 121 (1998), 1603-630: 1621.

it redressed the century-long therapeutic nihilism that patients with phantom limb pain had faced. Indeed it is the therapeutic efficacy of his method that has done most to cement Ramachandran's reputation, especially in popular circles. His experimental treatment for upper-arm amputees has been featured prominently in his best-selling book, Phantoms in the Brain (1998), and in the BBC 4 special programme of the same name.

Take the example of D.S., a man of 38 years, who had suffered a brachial plexus avulsion a decade before he came to see Ramachandran, and had had his arm amputated 6 inches above the elbow a year after the accident. He experienced pain at his phantom elbow several times every day. Moreover, he experienced his phantom arm as 'frozen', and felt completely unable to move it, which bothered him significantly. On appearing at Ramachandran's office, D.S. was asked to place his arm in the mirror box. D.S. experienced substantial difficulty achieving this (patients with moving phantoms usually found this easy; those with paralysed phantoms could do it by lifting up their shoulder thus dragging the phantom into the box), but once the phantom was in place, he could 'see' his paralysed arm moving. D.S. exclaimed in surprise: 'This is mind-boggling. My arm is plugged in again; it's as if I am back in the past ... Doctor, [my arm] no longer feels like it's lying lifeless in a sling.' 23

Another relatively common phenomenon was a 'clenching spasm', an involuntary contraction of the phantom hand, which patients to their great annoyance could usually only unclench with difficulty. The spasm could be painful because patients would often feel their phantom 'fingernails digging into the palm'. ${ }^{24}$ Patient R.T., a 55-year old engineer, was one example of a patient suffering from clenching spasm. Under normal conditions, it would take him at least half an hour to unclench his phantom hand, but when the illusion that his phantom arm had been brought to life again was produced in the mirror

${ }^{23}$ Ramachandran and Rogers-Ramachandran, op. cit. (note 1), 381.

${ }^{24}$ Ibid., 380. 
box, he exclaimed with surprise that 'all his movements had come back' and that he 'vividly experienced muscle and joint movements in his phantom'. ${ }^{25} \mathrm{He}$ could unclench his phantom with ease. The spasm had disappeared completely.

\section{How Did the Mirrors Work?}

To understand how the mirrors were supposed to work, we need to turn to Ramachandran's earlier interest in visual perception and ambiguities. In a 1986 paper, Ramachandran and the psychologist Stuart Anstis observed that the brain filled in gaps between multiple still images of a certain kind, and thus perceived movement, what Ramachandran and Anstis called 'apparent motion'. It is the same phenomenon film producers use to create the illusion of a 'moving picture'. ${ }^{26}$ For instance, if a dot is presented in one frame, and in the next frame an identical dot is positioned slightly to the side, the viewer will perceive the dot as moving, even though all the constituent parts of the phenomenon are static.

Researchers had been aware of this process for some time, but it was the mechanism that Ramachandran presented that was new, a mechanism whose value only became clear when dealing with highly complex images. Take the example of a leopard jumping from one tree to another. In traditional explanations, drawing on a computer model, the visual system would process the images as assemblages of tiny points of varying brightness. Each point in one frame would then be compared to points in the next frame to give the impression of a single moving body. But for Ramachandran and Anstis this explanation would require the brain to have access to massive computing power, and was implausible as a model for the way in which it worked. Instead they explained apparent motion by suggesting that it was 'controlled in the early stage of visual processing by what is in effect a bag of tricks, one the human visual system has acquired through natural selection during millions of years of evolution'. ${ }^{27}$ For one thing, this meant that the brain relied on the extraction of salient features such as brightness and texture that would help detect correspondence between an ensemble of different objects, like the dots. ${ }^{28}$ Most importantly, however, the researchers believed that 'the visual system assume[d] the world ha[d] order' ${ }^{29}$ The visual system relied on a set of schemata, or 'built-in laws of motion' when processing the visual features. ${ }^{30}$ For example, it would perceive linear motion in preference to abrupt changes in direction - a law that, as Ramachandran and Anstis noted, was 'reminiscent of Isaac Newton's first law of motion, namely that objects in motion tend to continue their motion along a straight path' ${ }^{31}$ Or the visual system would observe the principle of rigidity according to which 'all points on a moving object [were] assumed to move in synchrony'. ${ }^{32}$ These principles, in one way or another, 'reflect[ed] a built-in knowledge of properties of

\footnotetext{
25 Ibid.

26 V.S. Ramachandran and Stuart M. Anstis, 'The Perception of Apparent Motion', Scientific American 254, 6 (1986),102-9.

${ }^{27}$ Ramachandran and Anstis, op. cit. (note 26), 102.

${ }^{28}$ Note that this brightness is what is called 'coarse brightness' or 'low spatial frequency brightness', which is the converse of 'pixel brightness' or 'high spatial frequency brightness'. The latter would not be a useful cue because it would require excessive computational power.

${ }^{29}$ Ramachandran and Anstis, op. cit. (note 26), 105. In fact, perceptual scientists find the visual system to be remarkably attuned to statistical regularities that occur in the natural world. For a review, see W.S. Geisler et al., 'Natural Systems Analysis', Visual Neuroscience, 26 (2009), 1-3.

${ }^{30}$ Ramachandran and Anstis, op. cit. (note 26), 109.

${ }^{31}$ Ibid., 105 .

32 Ibid., 106.
} 
the physical world'. ${ }^{33}$ In the case of the moving leopard, one such assumption would be that the spots 'move[d] in synchrony with the salient feature', that is, the leopard itself. ${ }^{34}$ The brain did not infer the movement of a number of moving spots independently and then combine them to see a leopard jumping. Rather it saw a leopard jumping, assuming all the spots would move in formation. Ramachandran's model thus relied on a number of schemata, gained through experience either individually or as a species, that helped the brain make sense of and process raw visual input. Perception was possible because sense impressions were ordered in the brain according to a pre-set, but not entirely immutable, set of laws. In other words, perceptual experience was organised around expectations.

In Ramachandran's model of perception we see the positing of two parallel realms. The brain interpreted sense data by drawing on a set of rules that were supposed to mirror those of the real world. Successful activity was dependent upon the match of the mental schemata and physical laws. In Ramachandran's understanding of the phantom limb phenomenon, we see the displacement of this opposition between two separate but parallel orders onto an immaterial phantom and a physical body. In our normal experience of our bodies, the sense impressions coming from our various limbs, whether from touch, the feeling of movement, or visual stimuli, were ordered around a number of expectations. For instance if we wanted to flex our arm, a signal would be sent from the motor cortices to the biceps muscle. At the same time, a copy of the signal ('efferent copy') would be sent to parietal cortices for forward prediction of the imminent sensory state of the biceps, termed 're-afference'. The predicted re-afferent signal would contribute to what Ramachandran called a 'dynamic body image', an internal impression of the moving arm and its sensory consequences. ${ }^{35}$ Such a body image functioned like the 'built-in laws of motion' of perception. It provided an ordering expectation of the subsequent sensory impressions. Just as we would assume that the leopard's spots maintained a consistent pattern as it jumped through space, so too the dynamic body image created the expectation of sensory impressions from the elbow joint flexing, and the visual image of our lower arm rising. It was an entity, produced by a copy of a motor command, which created the expectation of an actual movement. As Ramachandran pointed out, in the parietal lobe, the dynamic body image was then compared to the actual sense impressions. ${ }^{36}$

The co-existence of these two bodies, the internal dynamic body image and the external physical body provided a framework for explaining phantom limbs. According to Ramachandran, when 'a central representation of the limb survives after amputation', the mismatch between the body image and the real body was 'largely responsible for the illusion of a phantom'. ${ }^{37}$ The schemata persisted even as the limb disappeared, and were so powerful that they strongly resisted the evidence of sense impressions that suggested that the body part was no longer there. ${ }^{38}$ In this case, we can say, the internal, 'imagined' expectation was lent greater credibility than the external, 'real' movement. But the dominance of image over reality was not total. Rather the characteristics of the phantom limb were structured by its relationship to the real body. D.S. considered his

33 Ibid., 102.

${ }^{34}$ Ibid., 106.

${ }^{35}$ For example, in Ramachandran et al., op. cit. (note 8).

36 Ibid., 30.

37 V.S. Ramachandran and William Hirstein, 'The Perception of Phantom Limbs: The D.O. Hebb Lecture', Brain, 121 (1998), 1603-630: 1604.

${ }^{38}$ V. S. Ramachandran, 'Phantom limbs, neglect syndromes, repressed memories, and Freudian psychology', International Review of Neurobiology, 37 (1994), 291-333: 317. 
phantom limb to be paralysed because despite his attempts to move it, sending out a motor command from his motor brain map, he received no proprioceptive or visual evidence that it had changed position. As Ramachandran wrote: 'Eventually, the brain learns that the arm does not move and a kind of "learned paralysis" is stamped onto the brain's circuitry. ${ }^{39}$ So too R.T. felt pain in his hand because without visual evidence he found it difficult to convince himself that his phantom hand could be unclenched.

Given this analysis of the phantom limb phenomenon, we can see how Ramachandran might have understood the efficacy of the mirror box. The mirror created its own dynamic representation, a 'virtual' image, of the physical body. The reflection of the right healthy hand (for instance) was deployed in such a way that it could be understood by the patient to be her left hand. To achieve this, the patient had to replicate with the right hand the felt movement or position of the phantom left hand in such a way that the mirror image and the internal dynamic body image aligned. In this way the mirror 'restore[d] the visual feedback in response to the motor command', and created the illusion of wholeness. ${ }^{40}$

Under these conditions, the brain was no longer convinced of paralysis, because it saw the hand moving in unison with the motor commands asking it to move. Moreover, pain caused by clenching could be solved, because the compatibility between what was seen and the body image allowed the patient greater control over the movement of the phantom limb. When the brain sent a signal for the hand to become unclenched, it could now see the hand unclench itself, convincing itself that this had actually happened, and thus causing the pain to disappear.

And though the chain of relationships is long and complex - the right healthy hand reproducing the movements of the phantom, the mirror producing an immaterial reflection of that movement, the patient taking the immaterial mirror image to be the real physical left hand, which was consequently compared to the phantom - at its most basic level, the mirror created an immaterial double within the experiment that could be aligned with the 'body image' in the brain. Indeed, like the dynamic body image, the mirror image was both a careful reproduction of the real body and crucially out of sync with it; the mirror produced its own phantoms. And this is why it was the central element of Ramachandran's experimental apparatus: the mirror allowed him to manipulate and thus experiment on the phantom limb. Only the mirror made the phantom present, malleable, open to study.

What happened in this meeting of mirror and phantom was unclear. Was it the return of a prodigal son, the phantom finally making its way to the land of neuroscientific respectability, revealing itself to be subject to the same laws as other scientific objects? That is, was Ramachandran's mirror phantom no longer really a phantom? Or did it rather mark a movement in the other direction, where the phantom revealed the mirror to be its own form of occult instrument? For the mirror was a meeting point between the real and the virtual, the body and its phantom, the material and the immaterial, a conjuncture between different ontological spheres.

\section{Plasticity}

This understanding of the mirror box could only explain short-term respite from phantom limb pain. Sure enough, in the case of D.S., when the mirror was removed, the sensation disappeared, and D.S felt his limb to be 'frozen again'. So too R.T.'s hand remained

\footnotetext{
39 V.S. Ramachandran, with Sandra Blakeslee, Phantoms in the Brain: Probing the Mysteries of the Human Mind (New York: Harper Perennial, 1998), 46.

${ }^{40}$ Ramachandran and Altschuler, op. cit. (note 22), 1696.
} 
unclenched only for a few hours, until the next spontaneous cramp occurred. But Ramachandran wondered if the change could be made permanent. He gave D.S. the mirror box to take home, and instructed him to practice for 15 minutes a day for several weeks. Three weeks later, the phone rang in Ramachandran's office. D.S. reported that his phantom arm had now disappeared. All that was left were his fingers and part of the palm 'dangling from the stump near the shoulder', a phenomenon that Ramachandran called 'telescoping'. ${ }^{41}$ The patient welcomed the telescoping because it had made the phantom elbow, and thus the pain located there, disappear. Ramachandran jokingly referred to the case as the "first known case of an "amputation" of a phantom limb'. ${ }^{42}$

Ramachandran's explanation for this development relied on a process that had played a small role in the analysis of phantom limbs in the first place. For though the phantom limb created a mismatch between expectation and sensation, it was not entirely devoid of sensory evidence. First, the re-afference signal which contributed to the dynamic body image remained even in an amputee. The parietal lobe continued to receive indications that the limb had been asked to move. Second, Ramachandran conceded that 'neuromas' - growths of nerves at the stump that were damaged at amputation and continued to send signals to the brain - might play a role. ${ }^{43}$ Third, and most important for Ramachandran, was the 'spontaneous activity of tissues in the face and tissues proximal to the amputation'. With this, Ramachandran entered into a discussion about neuroplasticity.

Thirty years earlier the dogma that the brain was immutable had begun to be challenged. In its stead, neuroscientists began to believe that the brain underwent changes over time, in response to environmental changes, mental processes, and physical injury. ${ }^{44}$ The precise mechanism of these changes was not yet fully clear. For Ramachandran, however, plasticity became significant through a process that he called 're-mapping'. ${ }^{45} \mathrm{Re}$-mapping, of which the exact workings still had to be determined (Ramachandran postulated that it could occur through the unmasking of silent synapses, or through anatomical sprouting), was dependent on pre-existing brain maps, such as Wilder Penfield's somato-sensory homunculus. In the homunculus, an illustration of the somato-sensory representation of the body on the post-central strip of the cortex, the hand area was framed by the face on the one side, and by the upper arm on the other side. When the arm was lost, the vast majority of stimuli normally received by that part of the cortex disappeared too. The lack of signals to that part of the brain made it particularly sensitive to signals passing through proximate areas. For that reason, stimuli sent to the neighbouring part of the cortex (dedicated to the face) might now be felt by the otherwise inactive part dedicated to the arm and hand; the patient would experience sensations on the face as sensations on the arm and hand. In this way the parietal lobe continued to receive evidence that the arm still existed, and thus refused to abandon or modify the pre-existing body image.

${ }^{41}$ Ramachandran and Rogers-Ramachadran, op. cit. (note 1), 382.

42 Ramachandran and Rogers-Ramachadran, op. cit. (note 1), 382, 386.

43 Such persistence also helped explain the phenomenon of neglect, which Ramachandran thought of as the 'converse of the phantom limb experience'. Here patients would refuse to acknowledge a paralysed limb, because the reality of the body image in the parietal lobe trumped the evidence that an arm, for example, could not move. Ramachandran, op. cit. (note 38), 314.

${ }^{44}$ For a scholarly discussion of neuroplasticity, see Nikolas Rose and Joelle Abi-Rached, Neuro: The New Brain Sciences and the Management of the Mind (Princeton, NJ: Princeton University Press, 2013), especially chs 1 and 5, and the work by Tobias Rees, for example, On How Adult Cerebral Plasticity Research Has Decoupled Pathology from Death, in David Bates, and Nima Bassiri (eds), Plasticity and Pathology: On the Formation of the Neural Subject (New York: Fordham University Press, 2016), 309-341.

${ }^{45}$ Ramachandran, 'Phantom limbs', 314 . 


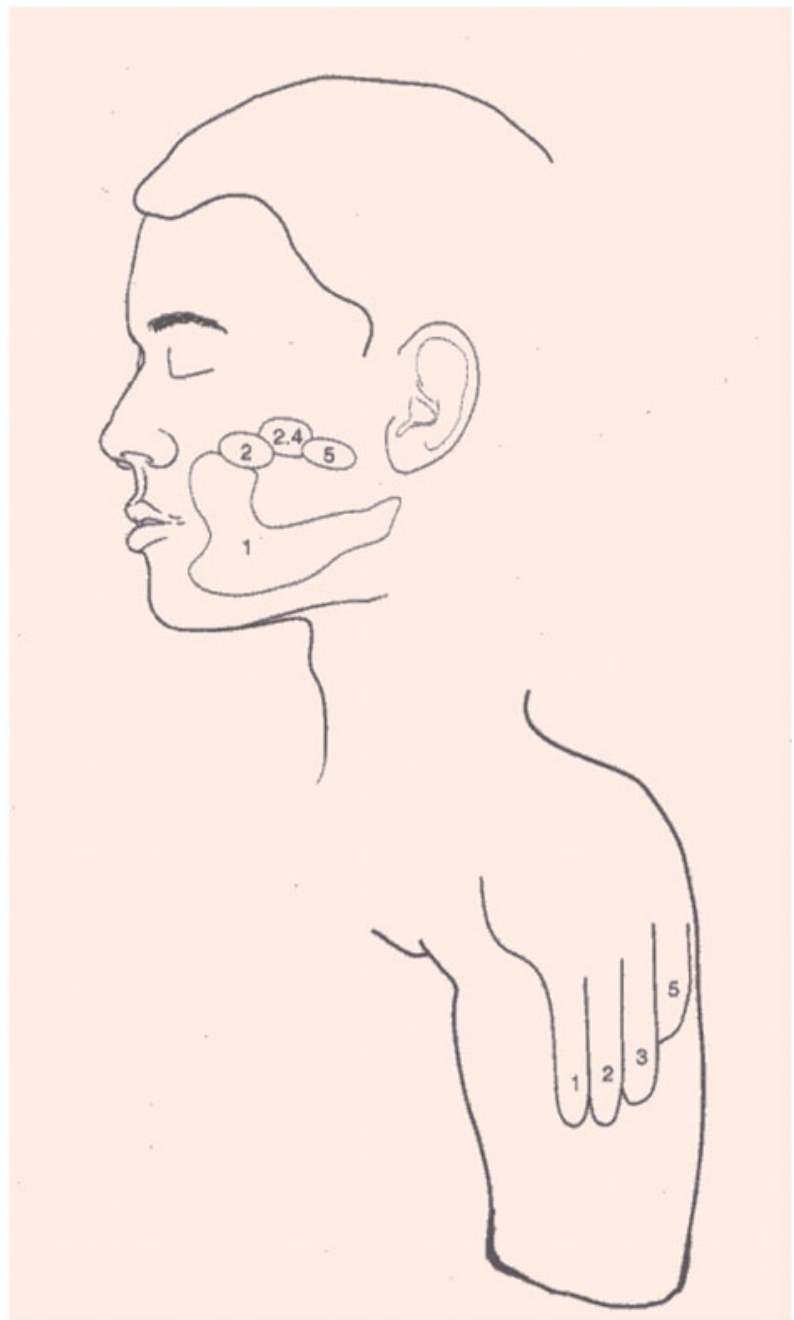

Figure 2: Phantom maps, face and arm. V.S. Ramachandran and Eric Altschuler, 'The Use of Visual Feedback, in particular Mirror Visual Feedback, in Restoring Brain Function', Brain, 132 (2009), 1693-1710: 1695.

Ramachandran demonstrated the existence and nature of re-mapping in an experiment about the localisation of touch. Stimuli were applied to a group of patients using a cotton swab at random points over the subject's bodily surface, and patients were asked to report where they felt the sensation. Some patients displayed the curious phenomenon of experiencing the touch on their amputated arm, in addition to the point where the touch was in reality applied. Figure 2 indicates the distribution in a particular amputee. ${ }^{46}$ Other patients located stimuli applied to the skin area above the amputation line on their phantom arms, providing information about the location of each finger. ${ }^{47}$ These 'phantom maps' could develop rapidly in newly amputated patients, sometimes over the course of weeks,

\footnotetext{
46 Ramachandran and Altschuler, op. cit. (note 22), 1695.

47 Apart from the fourth finger in the example.
} 
which demonstrated the plasticity of map building. As Ramachandran wrote, 'there [was] a tendency toward the spontaneous emergence of multiple somatotopically organised maps even in regions remote from the line of amputation'. ${ }^{48}$

Ramachandran subsequently confirmed these results in a magnetoencephalography (MEG) study. He and his co-workers mapped out the somato-sensory cortex of a patient whose arm had been amputated three inches below the elbow about ten years prior to the study. They took recordings from both hemispheres and, comparing them, found that the maps showed 'a striking asymmetry' caused, presumably, by the reorganised pathways in the left hemisphere. Most importantly, the 'hand' area in the left hemisphere was no longer visible and could be activated through touch on the newly mapped skin areas of the face and the arm above the line of amputation. ${ }^{49}$

The 're-mapping' of the body image indicated that it was to a certain extent plastic. Perhaps then this plasticity, which explained the phantom, could be deployed to get rid of it. As Ramachandran argued, the disappearance of D.S.'s phantom arm (and most of the associated pain) after two weeks of practice with the mirror box was the result of the 'longterm cortical reorganisation of brain maps' ${ }^{50}$ The repeated visual cues seemed to have an effect on the map itself, allowing it slowly to shift. In these experiments on re-mapping, Ramachandran relied on the assumption that, with the appropriate visual input, the body image was malleable; if the mirror image was shaped in just the right way, the phantom symptoms could eventually disappear. Indeed in a 2009 conference paper, Ramachandran suggested that the combination of lenses and mirrors could produce a gradually shrinking mirror image that would cause the phantom limb itself to shrink over time, curing the pain associated with it. ${ }^{51}$ Manipulation of the mirror image allowed the manipulation of the body image.

\section{Mirrors in the Brain}

Quite how visual stimuli were able to effect this re-mapping, however, remained an open question. In the short-term case, the parietal lobe simply compared the visual evidence with the body image. But long-term changes seemed to suggest that visual evidence played a more active role, that it could actually shape the map with which it was compared. How was it that visual input could refigure body maps? As Ramachandran's work developed in the late 1990s he speculated on a mechanism, and again mirrors were at the centre of his interest. This time, however, he found them within the brain.

In following this line, Ramachandran picked up on work from the early 1990s of a group of neuroscientists at the University of Parma, led by Giacomo Rizzolatti. The researchers were interested in area F5, located in the inferior premotor cortex, an area populated by neurons that became active during particular goal-directed hand movements, such as

\footnotetext{
${ }^{48}$ Ramachandran, op. cit. (note 38), 295.

49 V.S. Ramachandran, 'Behavioral and Magnetoencephalographic Correlates of Plasticity in the Adult Human Brain', Proceedings of the National Academy of the United States of America, 90, 22 (1993): 10413-20: 10418.

${ }^{50}$ Ramachandran and Altschuler, op. cit. (note 22), 1698.

51 V.S. Ramachandran et al., 'Shrinking Phantom Pain with Lenses and Shifting Referred Sensations through Volition', Neuroscience Abstracts (2009).
} 
grasping or holding, or for different kinds of hand grip. ${ }^{52}$ To investigate this region, Rizzolatti and his team recorded single neuron activity in macaque monkeys, who had been trained to manipulate objects of different shapes and sizes in a testing box. But while the neurons fired as expected when the monkeys performed the requisite tasks, they also fired at other times, when the monkeys weren't moving. As the experiment continued, it became clear that the neurons were activated when the monkeys simply observed others (either the experimenters or other monkeys) doing the same things. In his 1992 paper, Rizzolatti described these simply as a 'surprising new class of premotor neurons', but it was not long before they became known by another name: 'mirror neurons'. 53

Ramachandran's research into mirror neurons clearly followed from his earlier mirror work. To begin with, a common vocabulary informs both projects. In explaining Rizzolatti's mirror neuron system, Ramachandran described mirror neurons in a way that recalled his discussion of the mirror box. As we saw the mirror box was also referred to as a virtual reality box, because it allowed the production of a 'virtual' limb to stand in for the lost one. So too mirror neurons produced their own 'virtual realities', simulations of the other's action. ${ }^{54}$ More broadly, Ramachandran held that 'anytime you make a judgment about someone else's movements, you have to run a virtual-reality simulation of the corresponding movements in your own brain'. He added: 'And without mirror neurons, you cannot do this. ${ }^{55}$

So too, Ramachandran used the same kinds of patients to prove the existence of these neurons. One, a man named Humphrey, had lost his hand in the first Gulf War and had experienced a phantom ever since. Like other upper limb amputees he felt sensations in his hand when his face was touched. But he could also feel things that were done to someone else's hand. In an experiment, Ramachandran 'stroked and tapped' the hand of his student,

${ }^{52}$ G. Di Pellegrino et al., 'Understanding Motor Events: A Neurophysiological Study', Experimental Brain Research, 91 (1992),176-80: 176. For scholarly work on mirror neurons, see also Allan Young, 'The Social Brain and the Myth of Empathy', Science in Context, 25, 3 (2012), 401-24; Allan Young, 'Mirror neurons and the rationality problem', in S. Watanabe et al. (eds), Rational Animals, Irrational Humans (Tokyo: Keio University Press, 2009), 67-80; Susan Lanzoni, 'Imaging Emotions: Reconfiguring the Social in Neuroscience', paper presented at the annual meeting of the American Association for the History of Medicine, 8-11 May 2014; Katja Guenther, 'Imperfect reflections: norms, pathology, and difference in mirror neuron research', in David Bates and Nima Bassiri (eds), Pathology and Plasticity: On the Formation of the Neural Subject, Berkeley Forum in the Humanities (New York: Fordham University Press, 2016), 268-308. For a discussion of mirror neuron research from within the field, see Gregory Hickok, The Myth of Mirror Neurons: The Real Neuroscience of Communication and Cognition (New York: Norton, 2014).

${ }^{53}$ Di Pellegrino et al., op. cit. (note 52), 176. Two 1996 papers introduced the term 'mirror neuron'. Another early paper that Ramachandran cites is, M.S.A.Graziano et al., Science 226 (1994), 1051-4: V.S. Ramachandran and Diane Rogers-Ramachandran, 'Denial of Disabilities in Anosognosia', Nature, 382 (1996), 501. Mirror neurons were later used to describe the system's capacity for emotional empathy, a move criticised by Ruth Leys, " "Both of Us Disgusted in My Insula": mirror-neuron theory and emotional empathy', in Frank Biess and Daniel M. Gross (eds), Science and Emotions after 1945 (2014), 67-95; an earlier version of the paper appeared at nonsite.org.

54 V.S. Ramachandran, 'Sensations Referred to a Patient's Phantom Arm from Another Subject's Intact Arm: Perceptual Correlates of Mirror Neurons', Medical Hypotheses, 70, 6 (2008), 1233-4: 1233. See also his 2000 Edge article where he first calls the action of mirror neurons a 'virtual reality simulation', V.S. Ramachandran, 'Mirror Neurons and Imitation Learning as the Driving Force behind "The Great Leap Forward" in Human Evolution', Edge 2000 (http://edge.org/3rd_culture/ramachandran/ramachandran_p1.html), and an article in 2009: V.S. Ramachandran and David Brain, 'Sensations Evoked in Patients with Amputation from Watching an Individual whose Corresponding Intact Limb Is Being Touched', Archives of Neurology, 66 (2009), 1281-4: 1281.

55 See also V.S. Ramachandran, The Tell-Tale Brain: A Neuroscientist's Quest for What Makes us Human (New York: Norton, 2011), 123. 
Julie, and Humphrey experienced precisely the same kind of touch on his own phantom hand. Ramachandran explained the phenomenon in terms of mirror neurons, a group of which was responsible for the recognition of touch and pain. Just as in any other person observing someone else's hand being touched, Humphrey's touch mirror system became active. In healthy subjects, we don't perceive our hand as being stimulated because a lack of real input to our hand overrides the mirror system and cancels out the sensation of touch. But because Humphrey did not receive any disconfirming information from the phantom hand that the skin had not actually been touched, the evidence from the mirror system was taken at face value. As Ramachandran put it: 'there was no longer a null signal from the hand to veto' so that 'Humphrey's mirror neuron activity was emerging fully into conscious experience. ${ }^{56}$

Finally, Ramachandran increasingly saw mirror neurons as providing the key to unlocking the secrets of the mirror box treatment. Mirror neurons were fascinating for Ramachandran because they provided a mechanism by which visual input could be interpreted as proprioceptive or tactile input. As Ramachandran stated, 'mirror neurons necessarily involve interactions between multiple modalities - vision, motor commands, proprioception', and that is why they could explain the 'efficacy of MVF [mirror visual feedback ${ }^{57}$ : patients saw their paralysed phantom limbs move, and as a result they could feel them move as well. The mirror neurons thus explained the interchange between visual input and the dynamic body image that lay behind the phantom limb phenomenon. Moreover, Ramachandran argued that mirror neurons could explain how vision was able to reshift the map. Visual input to the mirror neurons could stimulate motor neurons on the body map that had been left 'inhibited' or 'dormant' after the stroke or amputation, recruiting them for new purposes. ${ }^{58}$ That is the existence of mirror neurons opened up a way to access neural material that had lost the possibility of direct sensory stimulation.

In his explanation then, Ramachandran posited a close relationship between the body maps that lay behind the phantom limb phenomena and mirror neurons. The virtual reality produced by the mirror neurons, an imaginative copying of the action in the brain, thus resembled the dynamic body image. After all, the maps and body images responsible for phantom limbs were themselves mirror-images (if sometimes distorted), that is, doubles of the moving body. The similarity explained why the 'virtual realities' produced by mirror neurons could help mould the body image. What is striking about Ramachandran's discussion of mirror neurons is that they occupy a similar structural position within the brain to his mirror box outside it. In both cases the mirror is capable of producing a virtual world. Ramachandran's argument approaches the claim that the body image is a mirror image too. In this way, by the end of the 1990s the mirror had, both as a concrete object and as a metaphor, colonised large swathes of Ramachandran's work. ${ }^{59}$

As Ramachandran's work developed, mirrors became ever more central. In a 2007 article tellingly titled, 'It's all done with mirrors' ${ }^{60}$ Ramachandran drew on mirror neurons to deal with evolutionary questions. Mirror neurons were, he argued, the driving force behind 'the great leap forward' in human evolution, the 'sudden explosion ...

\footnotetext{
56 Ramachandran, op. cit. (note 55), 125.

57 Ramachandran and Altschuler, op. cit. (note 22), 1702.

58 Ibid.

${ }^{59}$ See also Ramachandran et al., op. cit. (note 51).

${ }^{60}$ V.S. Ramachandran and D. Rogers-Ramachandran, Its All Done with Mirrors: Reflections on the familiar and yet deeply enigmatic nature of the looking glass, Scientific American Mind, August/September 2007, 16-18
} 
in technological sophistication, cave art, clothes, stereotyped dwellings, etc. around 40 thousand years ago'. ${ }^{61}$ Ramachandran even speculated that mirror neurons played a role in introspection and 'self-consciousness'. According to this argument, such neurons might function as the brain's 'internal mirror' ${ }^{62}$ Because for Ramachandran the mirror neuron became what made us human, it could be used to understand a wide range of phenomena, perhaps most controversially autism. Based on the observation that 'mirror neurons appear to be performing precisely the same functions that seem to be disrupted in autism', such as miming and imitating other people's actions, which in turn enabled social interaction, Ramachandran developed the hypothesis that autism consisted in a deficiency in the mirror neuron system: a 'broken mirror hypothesis'. Autistic children were not able to develop a 'theory of other minds' in the language of the Cambridge autism researchers Uta Frith and Simon Baron-Cohen, and the mirror neuron system for Ramachandran offered a mechanism to explain this failure. ${ }^{63}$ If 'broken mirrors' provided a theory of autism, Ramachandran speculated whether the 'mirrors [could] be repaired' ${ }^{64}$

What is remarkable in this development is the way Ramachandran's explanation of the phantom limb phenomenon, its genesis, structure and development, was increasingly marked by a vocabulary drawn from the material culture he used to study it. Phantoms were themselves distorted mirror images, just like the illusions created by the mirror boxes. And because the mirror emerged consistently in Ramachandran's work as a point of reference whenever his work broached the mind, we can think of it as a guiding metaphor for the mental tout court. The development is perhaps not surprising, for in the two key characteristics of the mirror that were at the heart of Ramachandran's mirror box practice - the ability to produce a faithful image, the ability to dissociate the image from what it reflected - we can see an outline of a fairly traditional theory of mind: a realm of representations which may or may not correspond to the real world. In the mirror, then, we see perhaps a way of approaching what has remained a conundrum for the materialist outlook of the neurosciences: how to understand the mind as a realm of representations.

\section{Freudian Reflections}

Ramachandran's mirror-based conceptualisation of the mind help us understand another aspect of his work. For his writing is haunted by another phantom: that of Sigmund Freud. The father of psychoanalysis is mentioned with insistent regularity throughout Ramachandran's articles, books and television appearances. We find reference to key Freudian concepts such as repression or the unconscious in his writings targeted at a popular audience, but also, and more surprisingly, Freud crops up continuously in Ramachandran's more narrowly scientific publications. ${ }^{65}$

Ramachandran's interest in Freud resembles his interest in phantom limbs: Freud's work was at first glance insufficiently somatic, and his ideas needed to be translated into rigorous neurological language in order to gain acceptance. Ramachandran was insistent

${ }^{61}$ Ramachandran, op. cit. (note 54).

62 Ramachandran and Rogers-Ramachandran, op. cit. (note 60), 16.

63 Baron-Cohen is, in fact, opposed to Ramachandran's theorisations.

64 V.S. Ramachandran and Lindsay M. Oberman, 'Broken Mirrors: A Theory of Autism', Scientific American, 295 (2006), 63-9.

${ }^{65}$ For example, throughout his Phantoms in the Brain - see op. cit. (note 39); throughout his recent Tell-Tale Brain - see op. cit. (note 55); in V.S. Ramachandran, A Brief Tour of Human Consciousness (New York: Pi Press, 2004), 1, 7-8; in op. cit. (note 8); in International Review of Neurobiology, (1994); and in Medical Hypotheses, (1996). 
that Freud 'was originally trained as a neurologist' and though for various reasons he had to depart from this path, Freud had 'never lost sight of his initial goal of providing a neural explanation for psychological phenomena'. ${ }^{66}$ It was up to Ramachandran to complete the task. To give his analyses legitimacy, Ramachandran simply had to 'anchor the airy abstractions of Freudian psychology in the physical flesh of the brain'. ${ }^{67}$

In his efforts to use psychoanalysis to inform modern neuroscience conceptually, Ramachandran did not stray too far from a group of neuroscientists, such as the Nobel laureate Eric Kandel and the neuropsychologist and historian of psychoanalysis Mark Solms, who had created the field of 'neuropsychoanalysis' (with its own journal of the same name in 1999). ${ }^{68}$ But Ramachandran's approach is interesting for us because he identified the cerebral body map as the central device for translating psychoanalysis into neuroscience. What Freud described as a psychological process was for Ramachandran a transformation at the level of the body image. Take for example Ramachandran's explanation of foot fetishes. He agreed with Freud that the foot could become symbolic of the penis, but Ramachandran linked the two through the proximity of the foot and genital area on the somato-sensory map. Body parts, whose cortical representation was further away from that of the genitals, as Ramachandran pointed out, were far less likely to be fetishised. To Ramachandran, the 'map-dominated' character of the human being offered a far more convincing explanation than Freud's psychodynamic explanation. ${ }^{69}$

Similarly, Ramachandran used the body image for thinking through the Freudian conception of repression and thus of the unconscious. Taking the example of his anosognosia patients (patients who were unaware of or denied their illness), Ramachandran asserted a 'striking similarity between the strategies these patients use and what Sigmund and Anna Freud called psychological defence mechanisms'. ${ }^{70}$ For Ramachandran, anosognosia and the repression it represented could be explained by the priority of the intact dynamic body image over the paralysed body. The absolute precedence of the body map in anosognosia patients required them to deny or 'repress' evidence to the contrary. ${ }^{71}$

That the body image was Ramachandran's central tool while grappling with Freud is telling. As we have seen, Ramachandran increasingly understood the body image as a type of mirror image, produced and tinkered with through the action of mirror-neurons. In this sense, Ramachandran's understanding of Freud, like his understanding of phantom limbs, is guided by a mirroring principle; once again for Ramachandran, the mirror sits at the border between mind and brain, this time in its disciplinary guise: the dividing line between psychoanalysis and neuroscience.

For Ramachandran, the mirror is unequivocally a materialist construction - mirrors could be explained by the laws of optics, there was not a ghost or phantom in sight. It helped make the phantom limb and Freudian analyses acceptable to and readable for somatic medicine. And yet, at times in his writing, Ramachandran admitted that the mirror remained 'deeply enigmatic'. ${ }^{72}$ Despite its materiality it produced a 'virtual reality', a non-material doubling of the real body, that looked and even acted like a phantom. And

\footnotetext{
66 Ramachandran et al., op. cit. (note 8), 29.

${ }^{67}$ Ramachandran et al., Ibid., 30.

${ }^{68}$ For a critique of 'neuro-psychoanalysis', see Nima Bassiri, 'Freud and the Matter of the Brain: On the Rearrangements of Neuropsychoanalysis', Critical Inquiry, 40, Autumn (2013), 1-26.

${ }^{69}$ Ramachandran, op. cit. (note 38), 316.

${ }^{70}$ Ramachandran et al., op. cit. (note 8), 39.

${ }^{71}$ Ramachandran et al., Ibid., 39-40.

72 Ramachandran and Rogers-Ramachandran, op. cit. (note 60), 16 .
} 
this virtual reality was not simply a faithful copy. Rather it could be, perhaps always was, slightly out of joint with reality. Indeed the disjuncture between reality and the mirror image was crucial for Ramachandran's work: therapeutically in the mirror box it provided the image of an arm that was not, in fact, there; conceptually in the role of mirror neurons, it stood in the liminal ground between mental action and real execution. For this reason, though the mirror-map was used by Ramachandran as a way of 'anchor[ing]' Freud's 'airy abstractions' in the 'physical flesh of the brain', it could also be interpreted in a diametrically opposed way. In relying so heavily on mirrors Ramachandran might not have bypassed the problems of the ambiguous and complex relationship between the psychological and the somatic, but rather have integrated that ambiguity into his somatic theory. For Ramachandran the cerebral self was a hall of mirrors, where the real and the unreal, the object and its image could never be absolutely and reliably distinguished.

Ramachandran was not alone in posing the self as a form of mirroring. Indeed the use of the mirror, both as a concrete object in experiments and as a metaphor in analysis, seems to cut across the wide variety of disciplines and orientations within the mind and brain sciences. ${ }^{73}$ It emerges at key moments in the history of psychoanalysis, developmental and clinical psychology, neurology, and modern neuroscience, as if these disciplines are irresistibly drawn to its strange mysteries. In all these contexts, the mirror is fascinating because it remains ambiguous. For the mirror is that strange impermeable and yet transparent boundary between the real and the imaginary, the physical and the psychological, the material and the immaterial, and when we see ourselves on the other side of the glass, we might wonder whether it also has the power to catch souls.

\footnotetext{
${ }^{73}$ Mirrors have been fascinating to many scholars although we still lack a history of the mirror within the sciences of the mind. See, for example, Mark Pendergrast, Mirror Mirror: A History of the Human Love Affair with Reflection (New York: Basic Books, 2003); Sabine Melchior-Bonnet, The Mirror: A History (New York: Routledge, 2001).
} 